\title{
New sociology for better understanding severe eosinophilic asthma: introducing the SOCS family
}

\author{
Delphine Gras ${ }^{1}$ and Pascal Chanez ${ }^{1,2,3}$
}

\begin{abstract}
Affiliations: ${ }^{1}$ UMR INSERM 1067/CNRS 7333, Marseille, France. ${ }^{2}$ Aix-Marseille Université, Marseille, France. ${ }^{3}$ APHM (Assistance Publique Hôpitaux de Marseille), Clinique des Bronches, de l'Allergie et du Sommeil, Hôpital Nord, Marseille, France.
\end{abstract}

Correspondence: Delphine Gras, UMR Inserm U1067 CNRS 7333, Hôpital de la Conception, 147 Bd Baille, Marseille 13005, France. E-mail: delphine.grasđuniv-amu.fr

SOCS proteins' involvement in severe eosinophilic asthma http://ow.ly/lG943023yxZ

Severe asthma is a heterogeneous condition characterised by a persistent uncontrolled situation with an inadequate response to current therapy [1]. This definition points the serious unmet needs of the patients and an urgent research effort to understand better the mechanisms sustaining this clinical condition for our community. However, severe asthma is still not identified as a distinct entity and is very heterogeneous. At present, several endotypes have been described based on the presence of upregulated cytokines leading to the recruitment, activation and increased survival of inflammatory cells such as eosinophils. One major endotype is related to T2 lymphocyte (T-helper cell type 2 and innate lymphoid cell type 2) activation, which is observed in most severe asthma patients. Fortunately, several new drugs are or have been developed to interfere with this endotype, but there are still important gaps of knowledge to be filled and clearly, other targets should be identified to develop innovative treatments [2].

The SOCS (suppressors of cytokine signalling) family is composed of eight proteins, i.e. SOCS1-7 and SH2 cytokine-inducible protein (CIS) [3, 4], each of which has a central SH2 domain, an amino-terminal domain of varying length and sequence, and a carboxy-terminal 40-amino-acid-long module known as the SOCS box [5]. SOCS proteins act as a substrate-recruiting component of E3-ubiquitin ligase complexes and target interacting proteins for degradation. The regulation exerted by SOCS proteins is critical to the normal functioning and cessation of the primary cytokine signal, and is achieved at many levels in the intracellular biochemical cascade [6]. Aberrant regulation of SOCS proteins has been linked to a variety of inflammatory diseases [7]. The most studied family members, CIS, SOCS1, SOCS2 and SOCS3, are important regulators of the Janus kinase (JAK)-signal transducer and activator of transcription (STAT) pathway, and SOCS1-3 have been studied in detail, including the development of knockout mice [8-10]. SOCS1 is a member of this cytokine signalling pathway inhibitor family and is induced by a variety of cytokines, including interferons (IFNs). It inhibits signalling by suppression of JAK kinase activity [11] and by a promotion of the degradation of the activated cytokine-receptor complex [12]. T2-associated cytokines, such as interleukin (IL) 4 or IL13, are major factors of the ongoing inflammation, including airway eosinophilia, found in severe asthma. Interestingly, they mediate their actions via the JAK-STAT pathway and are regulated by several proteins including the SOCS family.

Little information is available concerning the potential role of SOCS proteins in human asthma. A polymorphism in the SOCS1 promoter has been identified by HARADA et al. [13] in a Japanese population of adult asthma patients. This polymorphism correlates with asthma severity and with increased SOCS1 expression in nasal epithelial cells in vitro. An association between increased SOCS1 expression, allergic

Received: June 222016 | Accepted: June 222016

Conflict of interest: Disclosures can be found alongside this article at erj.ersjournals.com

Copyright (CERS 2016 
sensitisation and T2 polarisation has been subsequently reported [14]. Animal studies showed that SOCS1-deficient mice die within 3 weeks of age. This lethality is associated with the infiltration of many organs by macrophages and granulocytes, and with a profound depletion of lymphocytes $[15,16]$. A cross onto either an IFN- $\gamma$ - or a Stat6-null background is necessary to prolong survival, indicating that both IFN- $\gamma$-driven T1 and IL4-driven T2 responses may contribute to the rapid death [8, 17]. LEE et al. [18] have investigated the role of SOCS1 in regulating the asthma response using SOCS1 ${ }^{-1-} \mathrm{IFN}-\gamma^{-1-}$ mice in an acute ovalbumin-driven model of asthma. They reported elevated IgE levels, pathological evidence of eosinophilic lung infiltration and elevated T2 cytokine levels (IL4, IL5 and IL13). These results clearly indicate that SOCS1 is an important regulator of T2-type responses and in the presence of IFN- $\gamma$, SOCS1 would be involved in regulating both $\mathrm{T} 1$ and $\mathrm{T} 2$ responses [18]. In mice, SOCS2 negatively regulates T2 immunity [9], and SOCS3 mRNA levels of T-cells are increased in asthmatic patients and correlate with IgE levels [10]. Notwithstanding that, a functional role for SOCS3 in human asthma has not been described.

Interestingly, in this issue of the European Respiratory Journal, Doran et al. [19] investigated SOCS1-3 expression within the airways of subjects with asthma, and explored the relationship between SOCS expression and persistent eosinophilic inflammation within the airways. They reported decreased epithelial SOCS1 expression in asthmatics with persistent eosinophilia and increased T2 inflammation. These results corroborate previous findings in animal models. Moreover, they demonstrated that SOCS1 mRNA was significantly decreased in the airways of severe asthma patients when compared to mild/moderate asthma patients but that no difference was found when compared to healthy subjects. However, a recent study investigating SOCS1 and SOCS3 proteins in endobronchial biopsies showed that SOCS1, but not SOCS3, protein staining intensity was significantly increased in the bronchial epithelium of patients with asthma, as compared to healthy volunteers [20]. These observed differences could be partially explained by the fact that the data from Doran et al. [19] were obtained from whole endobronchial biopsy mRNA and, therefore, integrated several cell types, not exclusively epithelial cells. The mechanistic relationship between IL13 and SOCS1 signalling shown in vitro in an epithelial cell line would have been better justified after proving SOCS1 expression decreased specifically in epithelial cells from healthy and asthma patients. The discrepancies between studies should be acknowledged. The severity of the population studied is different (mild/moderate versus severe asthma), with a potential specific regulation of SOCS family proteins in severe asthma. Additionally, GIELEN et al. [20] found that SOCS1, but not SOCS3, mRNA expression levels are increased in primary human bronchial epithelial cells from children with severe asthma. This last finding points to the age of patients as a confounding factor, which may be crucial to understanding the biology of the SOCS proteins. Genomic single-nucleotide polymorphisms in the human SOCS1 gene were found to be associated with serum IgE levels [21]. Hence, an allergic status may be another important factor driving the expression of SOCS proteins irrespective of the age of the subjects or the severity of their asthma.

In their study, Doran et al. [19] confirmed that SOCS1 is a potent physiological negative regulator of T2 cytokines such as IL13. Moreover, SOCS1 expression was negatively related to hypereosinophilia and T2 gene signature. As shown in other studies, increased SOCS1 levels in epithelial cells from asthmatic patients impair type I and type III IFN induction suppressing antiviral pathways. This represents a novel mechanism explaining IFN deficiency in asthmatic patients [20]. Therefore, modulation of SOCS1 function is worthy of investigation as a new therapeutic approach.

In conclusion, the study presented by DoRAN et al. [19] highlights a novel role for SOCS proteins in severe eosinophilic asthma but their action is highly context dependent. Hence, it would be necessary to better define the physiological function of SOCS proteins by identifying the inducing stimuli, the relevant cell type, the SOCS substrate(s) and the biological context in which SOCS regulation is critical. SOCS proteins are potential new candidates for the treatment of asthma but their precise role in this disease should be clarified according to the different phenotypes and endotypes of asthma.

\section{References}

1 Chung KF, Wenzel SE, Brozek JL, et al. International ERS/ATS guidelines on definition, evaluation and treatment of severe asthma. Eur Respir J 2014; 43: 343-373.

2 Wenzel SE. Asthma phenotypes: the evolution from clinical to molecular approaches. Nat Med 2012; 18: 716-725.

3 Starr R, Willson TA, Viney EM, et al. A family of cytokine-inducible inhibitors of signalling. Nature 1997; 387: 917-921.

4 Elliott J, Johnston JA. SOCS: role in inflammation, allergy and homeostasis. Trends Immunol 2004; 25: 434-440.

5 Kubo M, Hanada T, Yoshimura A. Suppressors of cytokine signaling and immunity. Nat Immunol 2003; 4: 1169-1176.

6 Linossi EM, Babon JJ, Hilton DJ, et al. Suppression of cytokine signaling: the SOCS perspective. Cytokine Growth Factor Rev 2013; 24: 241-248.

7 Yoshimura A, Suzuki M, Sakaguchi R, et al. SOCS, inflammation, and autoimmunity. Front Immunol 2012; 3: 20x. 
Naka T, Tsutsui H, Fujimoto M, et al. SOCS-1/SSI-1-deficient NKT cells participate in severe hepatitis through dysregulated cross-talk inhibition of IFN- $\gamma$ and IL-4 signaling in vivo. Immunity 2001; 14: 535-545.

9 Knosp CA, Carroll HP, Elliott J, et al. SOCS2 regulates T helper type 2 differentiation and the generation of type 2 allergic responses. J Exp Med 2011; 208: 1523-1531.

10 Seki Y, Inoue H, Nagata N, et al. SOCS-3 regulates onset and maintenance of TH2-mediated allergic responses. Nat Med 2003; 9: 1047-1054

11 Endo TA, Masuhara M, Yokouchi M, et al. A new protein containing an SH2 domain that inhibits JAK kinases. Nature 1997; 387: 921-924.

12 Hanada T, Kinjyo I, Inagaki-Ohara K, et al. Negative regulation of cytokine signaling by CIS/SOCS family proteins and their roles in inflammatory diseases. Rev Physiol Biochem Pharmacol 2003; 149: 72-86.

13 Harada M, Nakashima K, Hirota T, et al. Functional polymorphism in the suppressor of cytokine signaling 1 gene associated with adult asthma. Am J Respir Cell Mol Biol 2007; 36: 491-496.

14 Daegelmann C, Herberth G, Röder S, et al. Association between suppressors of cytokine signalling, T-helper type 1/T-helper type 2 balance and allergic sensitization in children. Clin Exp Allergy J Br Soc Allergy Clin Immunol 2008; 38: 438-448.

15 Starr R, Metcalf D, Elefanty AG, et al. Liver degeneration and lymphoid deficiencies in mice lacking suppressor of cytokine signaling-1. Proc Natl Acad Sci 1998; 95: 14395-14399.

16 Metcalf D, Alexander WS, Elefanty AG, et al. Aberrant hematopoiesis in mice with inactivation of the gene encoding SOCS-1. Leukemia 1999; 13: 926-934.

17 Alexander WS, Starr R, Fenner JE, et al. SOCS1 is a critical inhibitor of interferon $\gamma$ signaling and prevents the potentially fatal neonatal actions of this cytokine. Cell 1999; 98: 597-608.

18 Lee C, Kolesnik TB, Caminschi I, et al. Suppressor of cytokine signaling 1 (SOCS1) is a physiological regulator of the asthma response. Clin Exp Allergy J Br Soc Allergy Clin Immunol 2009; 39: 897-907.

19 Doran E, Choy DF, Shikotra A, et al. Reduced epithelial suppressor of cytokine signalling 1 in severe eosinophilic asthma. Eur Respir J 2016; 48: 715-725.

20 Gielen V, Sykes A, Zhu J, et al. Increased nuclear suppressor of cytokine signaling 1 in asthmatic bronchial epithelium suppresses rhinovirus induction of innate interferons. J Allergy Clin Immunol 2015; 136: 177-188.e11.

21 Mostecki J, Cassel SL, Klimecki WT, et al. A SOCS-1 promoter variant is associated with total serum IgE levels. J Immunol 2011; 187: 2794-2802. 